\title{
Soft Interference Cancellation and Channel Estimation in Uncoded Asynchronous DS-CDMA System
}

\author{
Pei Xiao, Erik Ström \\ Communication Systems Group, Dept. of Signals and Systems \\ Chalmers University of Technology, SE-412 96, Göteborg, Sweden \\ phone: +46 31772 1768, fax: +46 317721748 \\ E-mail: pei.xiao@s2.chalmers.se, erik.strom@s2.chalmers.se
}

\begin{abstract}
The conventional interference cancellation receiver is subject to performance degradation due to incorrect decisions on interference subtracted from the received signal. This paper aims at deriving algorithms to improve the performance of interference cancellation and channel estimation in an uncoded asynchronous DS-CDMA system with orthogonal modulation. Two soft cancellation schemes, one based on maximum a posterior (MAP), the other based on nonlinear minimum mean square error (MMSE) criterion are presented and proved to be superior to the conventional PIC scheme with minor increase in complexity. Furthermore, the best system performance ( $2 \mathrm{~dB}$ gain in a 21-user system) is observed when the derived soft information is also used for channel estimation.
\end{abstract}

\section{INTRODUCTION}

The system under study is an uncoded asynchronous DSCDMA system with orthogonal signalling formats. The transmitted chip sequence from a particular user is the concatenation of one of $M$ possible Walsh sequences (representing the transmitted symbol) and a long scrambling code. The Walsh (Hadamard) code is employed for combining the advantages of spreading and coding to achieve improved performance for spread spectrum (CDMA) systems. Due to the orthogonality of its codewords, it allows for initial noncoherent detection when channel information is not available.

A CDMA cellular communication system is inherently interference limited. This is due to the difficulty of maintaining orthogonality on the reverse link between code channels used by independent mobile stations, which transmit asynchronously. This form of interference limits the uplink capacity severely. Very significant capacity gains can be achieved if multiuser interference can be reduced, or if joint detection of all users is employed. The aperiodic nature of the long scrambling codes employed in this work precludes the use of linear multiuser detection schemes like linear MMSE detector and decorrelator, etc. due to high computational complexity. Nonlinear cancellation algorithms for $M$-ary orthogonal modulation in DS-CDMA systems were proposed in several papers. For instance, parallel and successive interference cancellation were presented in $[1,2]$. The interference is estimated and subtracted from the received signal before detection is done.
Iterative schemes for demodulating $\mathrm{M}$-ary orthogonal signalling formats in DS-CDMA systems were proposed in $[3,4]$, using nonlinear MMSE and PIC, respectively. Timevarying Rayleigh fading channel is assumed in those papers, necessitating channel estimation for effective interference cancellation.

The conventional interference cancellation receiver is subject to performance degradation due to incorrect decisions on interference that are subtracted from the received signal. To prevent error propagation from the decision feedback, soft interference cancellation was proposed, e.g., in $[5,6]$ for convolutionally coded systems. In this case, the soft information is readily available from the soft-output channel decoder. For uncoded systems, the soft information has to be derived by some other means. This paper aims at deriving soft interference cancellation algorithms to improve the performance of an orthogonal modulated asynchronous DS-CDMA system without convolutional coding. We then further extend the use of derived soft information for channel estimation.

\section{System Model And PIC Algorithm}

Fig. 1 shows the signal path for the user $k$. The $k^{\text {th }}$ user $j^{\text {th }}$ transmitted symbol is denoted by $i_{k}(j) \in\{1,2, \cdots, M\}$, and is mapped into $\mathbf{w}_{k}(j) \in$ $\left\{\mathbf{w}_{1}, \mathbf{w}_{2}, \cdots, \mathbf{w}_{m}, \cdots, \mathbf{w}_{M}\right\}$, which is one of the $M$ orthogonal signal alternatives. The Walsh codeword $\mathbf{w}_{k}(j) \in$ $\{+1,-1\}^{M}$, is then repetition encoded into $\mathbf{s}_{k}(j) \in$ $\{+1,-1\}^{N}$ so that each bit of the Walsh codeword is spread (repetition coded) into $N_{c}=N / M$ chips, and each Walsh symbol is represented by $N$ chips. The Walsh sequence $\mathbf{s}_{k}(j)$ is then scrambled (randomized) by a scrambling code unique to each user to form the transmitted chip sequence $\mathbf{a}_{k}(j)=\mathbf{C}_{k}(j) \mathbf{s}_{k}(j) \in\{+1,-1\}^{N}$ where $\mathbf{C}_{k}(j) \in\{-1,0,+1\}^{N \times N}$ is a diagonal matrix whose diagonal elements correspond to the scrambling code for the $k^{t h}$ user's $j^{t h}$ symbol. The purpose of scrambling is to separate users. In this paper, we focus on the use of long codes, e.g., the scrambling code differs from symbol to symbol.

The baseband signal is formed by pulse amplitude modulating $\mathbf{a}_{k}(j)$ with a unit-energy rectangular chip waveform $\psi(t)$. The baseband signal is then multiplied with a carrier and transmitted over a Rayleigh fading channel with $L_{k}$ resolvable paths, having time-varying complex channel gains $h_{k, 1}(t), h_{k, 2}(t), \ldots, h_{k, L_{k}}(t)$ and delays $\tau_{k, 1}, \tau_{k, 2}, \ldots, \tau_{k, L_{k}}$. The received signal is the sum of $K$ users' signals plus additive white complex Gaussian noise $n(t)$. The received 


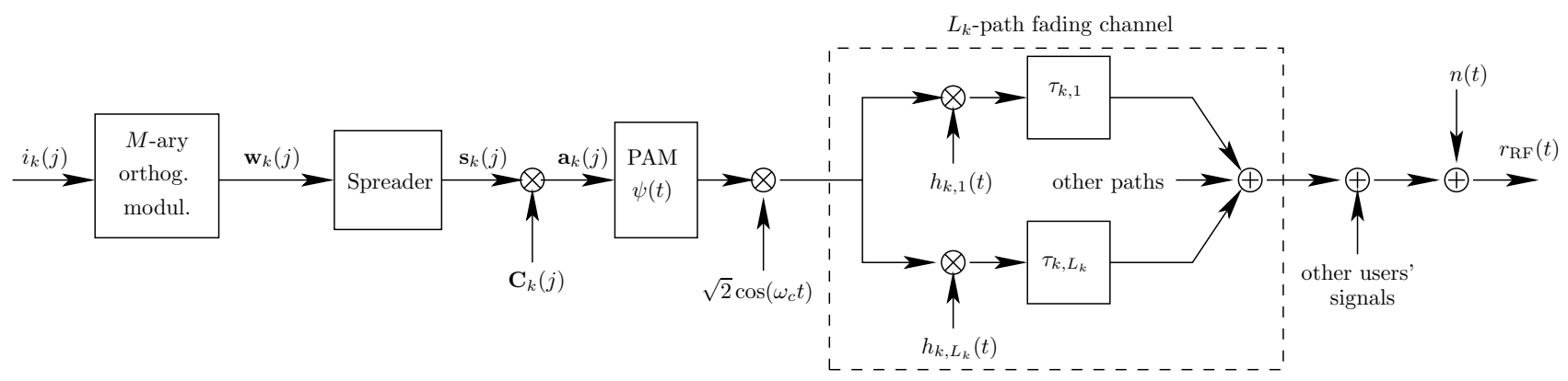

Fig. 1. Block diagram of the transmitter.

vector corresponding to the $k^{\text {th }}$ user's $j^{\text {th }}$ symbol after frequency down-conversion and chip matched filtering is denoted as $\mathbf{r}(k, j)$ and can be written in vector form as

$$
\begin{aligned}
& \mathbf{r}(k, j)=\mathbf{A}(k, j) \mathbf{h}(j)+\mathbf{n}(k, j) \\
& =\mathbf{X}_{k}(j) \mathbf{h}_{k}(j)+\operatorname{ISI}(\mathrm{k}, \mathrm{j})+\operatorname{MAI}(\mathrm{k}, \mathrm{j})+\mathbf{n}(\mathrm{k}, \mathrm{j}) \in \mathbb{C}^{\mathrm{N}_{\mathrm{k}}}
\end{aligned}
$$

where the columns of the matrix $\mathbf{A}(k, j)$ are essentially delayed versions of the transmitted chip sequences $\mathbf{a}_{k}(j)$ for $k=1,2, \cdots, K$, one column per path. The length of the processing window $N_{k}$, is larger than the symbol interval $N$ to account for the asynchronous and multipath nature of the channel. The columns are weighted together by $\mathbf{h}(j)$, whose elements are the path gains of all users' paths. The received vector $\mathbf{r}(k, j)$ can be written as the sum of four terms: the signal of interest $\mathbf{X}_{k}(j) \mathbf{h}_{k}(j)$, the intersymbol interference (ISI), the multiple access interference (MAI), and the noise represented by $\mathbf{n}(k, j)$, which is a vector of complex Gaussian noise samples with zero mean and variance $N_{0}$. The columns of the matrix $\mathbf{X}_{k}(j)$ are essentially the shifted versions of the chips due to the $k^{t h}$ user's $j^{\text {th }}$ symbol, one column per path (the shift is determined by the path delay). The vector

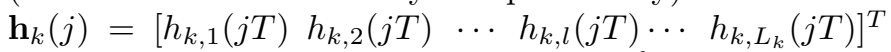
corresponds to the channel gains of the $k^{\text {th }}$ user's paths, it is a part of $\mathbf{h}(j)$.

The task of the receiver is to detect the information bits from all users, i.e., detect $i_{k}(j)$ for $k=1,2, \ldots, K$ given the observation $\mathbf{r}(k, j)$. The decision on the $k^{t h}$ user's $j^{\text {th }}$ symbol, is found as

$$
\hat{i}_{k}(j)=\arg \max _{m \in\{1,2, \cdots, M\}} z_{k}(m)
$$

where $z_{k}(m)$ is the decision statistic from symbol matched filter or multiuser detector (interference canceler in our case), based on the condition that the $m^{\text {th }}$ Walsh symbol is transmitted from user $k$. The conventional matched filter based single user receiver has poor performance in multiuser environment. Parallel interference cancellation (PIC) was proposed, e.g., in [3] to compensate for the effect of MAI. The basic principle is that once the transmitted signals are estimated for all the users at the previous iteration, interference can be removed by subtracting the estimated signals of the interfering users from the received signal $\mathbf{r}(k, j)$ to form a new signal vector $\mathbf{r}^{\prime}(k, j)$ for de- modulating the signal transmitted from user $k$, i.e.,

$$
\mathbf{r}^{\prime}(k, j)=\mathbf{r}(k, j)-\hat{\mathbf{A}}(k, j) \hat{\mathbf{h}}(j)+\hat{\mathbf{X}}_{k}(j) \hat{\mathbf{h}}_{k}(j)
$$

where $\mathbf{r}^{\prime}(k, j) \in \mathbb{C}^{N_{k}}$ denotes the interference canceled version of $\mathbf{r}(k, j)$ after subtracting the contributions from all the other users using decision feedback at the previous stage. The vector $\hat{\mathbf{A}}(k, j) \hat{\mathbf{h}}(j)$ represents the estimated contribution from all the users calculated by using the estimated data matrix $\hat{\mathbf{A}}(k, j)$ and channel vector $\hat{\mathbf{h}}(j)$. The vector $\hat{\mathbf{X}}_{k}(j) \hat{\mathbf{h}}_{k}(j)$ is the estimated contribution from all paths of user $k$. The soft decision with PIC is formed as

$$
z_{k}(m)=\operatorname{Re}\left\{\hat{\mathbf{h}}_{k}^{*}(j) \mathbf{X}_{k, m}^{*} \mathbf{r}^{\prime}(k, j)\right\}
$$

where $\mathbf{X}_{k, m}=\left[\begin{array}{llll}\mathbf{x}_{k, 1, m} & \mathbf{x}_{k, 2, m} & \cdots & \mathbf{x}_{k, L_{k}, m}\end{array}\right]$, and $\mathbf{x}_{k, l, m}$ denotes the transmitted chip sequence due to the $k^{\text {th }}$ user's $j^{t h}$ symbol from the $l^{t h}$ path based on the hypothesis that the $m^{\text {th }}$ Walsh symbol is transmitted.

\section{Soft Interference Cancellation}

In this section, we present soft PIC and channel estimation (CE) algorithms. The rationale is that the hard cancellation and $\mathrm{CE}$ tends to increase the interference and propagate errors with incorrect decision feedback; while with soft cancellation and $\mathrm{CE}$, the soft estimate of an erroneously estimated symbol usually has small value, and does not make much contribution to the feedback, therefore error propagation is avoided.

\section{A. Soft PIC based on MAP algorithm}

A posteriori log-likelihood ratio (LLR) for a transmitted +1 and a transmitted -1 in the bit sequence $\left\{\mathbf{w}_{k}^{n}(j)\right\}$ given received vector $\mathbf{r}(k, j)$ is defined as [7]

$$
\begin{aligned}
& \lambda\left(\mathbf{w}_{k}^{n}(j)\right)=\ln \frac{f\left(\mathbf{w}_{k}^{n}(j)=+1 \mid \mathbf{r}\right)}{f\left(\mathbf{w}_{k}^{n}(j)=-1 \mid \mathbf{r}\right)} \\
& =\ln \frac{f\left(\mathbf{r} \mid \mathbf{w}_{k}^{n}(j)=+1\right) P\left(\mathbf{w}_{k}^{n}(j)=+1\right)}{f\left(\mathbf{r} \mid \mathbf{w}_{k}^{n}(j)=-1\right) P\left(\mathbf{w}_{k}^{n}(j)=-1\right)}=\ln \frac{f\left(\mathbf{r} \mid \mathbf{w}_{k}^{n}(j)=+1\right)}{f\left(\mathbf{r} \mid \mathbf{w}_{k}^{n}(j)=-1\right)}
\end{aligned}
$$

$=\ln \frac{\sum_{m: \mathbf{w}_{k}^{n}(j)=+1} f\left(\mathbf{r} \mid \mathbf{w}_{m}\right)}{\sum_{m: \mathbf{w}_{k}^{n}(j)=-1} f\left(\mathbf{r} \mid \mathbf{w}_{m}\right)} \approx \ln \frac{\max _{m: \mathbf{w}_{k}^{n}(j)=+1} f\left(\mathbf{r} \mid \mathbf{w}_{m}\right)}{\max _{m: \mathbf{w}_{k}^{n}(j)=-1} f\left(\mathbf{r} \mid \mathbf{w}_{m}\right)}$

where $\mathbf{w}_{k}^{n}(j)$ denotes the $n^{\text {th }}$ bit of the codeword $\mathbf{w}_{k}(j)$. Equation (3) holds since bits +1 and -1 , are equally 
probable, i.e., $P\left(\mathbf{w}_{k}^{n}(j)=+1\right)=P\left(\mathbf{w}_{k}^{n}(j)=-1\right)$, for $n=1, \cdots, M$. We denote $m: \mathbf{w}_{k}^{n}(j)= \pm 1$ as the set of Walsh codes $\left\{\mathbf{w}_{m}\right\}$ that correspond to the code bit $\mathbf{w}_{k}^{n}(j)= \pm 1$. In the above equation, $\mathbf{r}$ can be replaced by its interference canceled version $\mathbf{r}_{k}^{\prime}$ for better performance. In case of perfect cancellation, $\mathbf{r}_{k}^{\prime}$ only contains the contribution from the $k^{\text {th }}$ user plus original additive Gaussian noise $\mathbf{n} \in \mathbb{C}^{N_{k}}$ with $\operatorname{PDF} \mathbf{n} \sim \mathcal{C N}\left(\mathbf{0}, N_{0} \mathbf{I}_{N_{k}}\right)$, i.e., $\mathbf{r}_{k}^{\prime}=\mathbf{X}_{k} \mathbf{h}_{k}+\mathbf{n}$. Therefore,

$$
\begin{aligned}
f\left(\mathbf{r}_{k}^{\prime} \mid \mathbf{w}_{m}\right) & =\frac{1}{\left(\pi N_{0}\right)^{N_{k}}} \exp \left(-\frac{\left\|\mathbf{r}_{k}^{\prime}-\mathbf{X}_{k, m} \mathbf{h}_{k}\right\|^{2}}{N_{0}}\right) \\
\lambda\left(\mathbf{w}_{k}^{n}\right) & \approx \ln \frac{\max _{m: \mathbf{w}_{k}^{n}=+1} f\left(\mathbf{r}_{k}^{\prime} \mid \mathbf{w}_{m}\right)}{\max _{m: \mathbf{w}_{k}^{n}=-1} f\left(\mathbf{r}_{k}^{\prime} \mid \mathbf{w}_{m}\right)} \\
& =\frac{2}{N_{0}} \operatorname{Re}\left\{\mathbf{h}_{k}^{*} \mathbf{X}^{+*} \mathbf{r}_{k}^{\prime}-\mathbf{h}_{k}^{*} \mathbf{X}^{-*} \mathbf{r}_{k}^{\prime}\right\}
\end{aligned}
$$

In (5), $\mathbf{X}^{+}$denotes the $\mathbf{X}_{k, m}$ that corresponds to $\max _{m: \mathbf{w}_{k}^{n}(j)=+1} f\left(\mathbf{r} \mid \mathbf{s}_{m}\right)$, and $\mathbf{X}^{-}$is defined similarly. Comparing (5) with (2), one can see that the added complexity by deriving soft values rather than making hard decisions is minor. Once the LLR value is derived, the soft estimate (expected value given the received observation) for each bit of the Walsh codeword can be computed as

$$
\begin{aligned}
\mathrm{E}\left[\mathbf{w}_{k}^{n}(j) \mid \mathbf{r}\right] & =(+1) \times P\left\{\mathbf{w}_{k}^{n}(j)=+1 \mid \mathbf{r}\right\} \\
& +(-1) \times P\left\{\mathbf{w}_{k}^{n}(j)=-1 \mid \mathbf{r}\right\} \\
& =(+1) \frac{e^{\lambda\left(\mathbf{w}_{k}^{n}(j)\right)}}{1+e^{\lambda\left(\mathbf{w}_{k}^{n}(j)\right)}}+(-1) \frac{e^{-\lambda\left(\mathbf{w}_{k}^{n}(j)\right)}}{1+e^{-\lambda\left(\mathbf{w}_{k}^{n}(j)\right)}} \\
& =\tanh \left\{\lambda\left(\mathbf{w}_{k}^{n}(j)\right) / 2\right\}
\end{aligned}
$$

The soft estimate $\mathrm{E}\left[\mathbf{s}_{k}^{q} \mid \mathbf{r}\right]$ for each Walsh chip $\mathbf{s}_{k}^{q}, q=$ $1, \cdots, N$ is derived by spreading (repetition encoding) the soft bit of Walsh codeword $\mathrm{E}\left[\mathbf{w}_{k}^{n}(j) \mid \mathbf{r}\right], n=1, \cdots, M$. The repetition factor is $N / M$. When $\mathrm{E}\left(\mathbf{s}_{k} \mid \mathbf{r}\right)$, the soft estimate of the transmitted sequence is available, we can carry out soft cancellation. The new vector after soft cancellation and decision statistic with MAP-PIC can be expressed as

$$
\begin{aligned}
& \mathbf{r}_{k}^{\prime}=\mathbf{r}-\mathrm{E}[\mathbf{y} \mid \mathbf{r}]+\mathrm{E}\left[\mathbf{X}_{k} \mid \mathbf{r}\right] \hat{\mathbf{h}}_{k} \\
& z_{k}^{\operatorname{MAP}}(m)=\operatorname{Re}\left\{\hat{\mathbf{h}}_{k}^{*} \mathbf{X}_{k, m}^{*} \mathbf{r}_{k}^{\prime}\right\}
\end{aligned}
$$

where $\mathrm{E}[\mathbf{y} \mid \mathbf{r}]=\mathrm{E}[\mathbf{A} \mid \mathbf{r}] \hat{\mathbf{h}}$, and the columns of $\mathrm{E}[\mathbf{A} \mid \mathbf{r}]$, $\mathrm{E}\left[\mathbf{X}_{k} \mid \mathbf{r}\right]$ are derived by scrambling $\mathrm{E}\left[\mathbf{s}_{k} \mid \mathbf{r}\right]$ with $\mathbf{C}_{k}$ and compensating with path delays.

\section{B. Soft PIC based on nonlinear MMSE estimation}

Nonlinear MMSE interference cancellation (NMIC) in DS-CDMA systems with BPSK and M-ary orthogonal modulation was proposed in [4], a multistage MMSE linear interference canceler that minimizes the power of residual cancellation error for each user was proposed to mitigate the effect of incorrect cancellation. The algorithm was originally derived for a single path asynchronous channel. Here, we extend its application to the multipath environments, and derive a logarithmic version of the NMIC algorithm which is suitable for practical implementation.
The composite received signal vector can be modeled as the sum of signals from different users, i.e., $\mathbf{r}=\mathbf{r}_{1}+\mathbf{r}_{2}+$ $\cdots+\mathbf{r}_{K}+\mathbf{n}$. Minimizing the residual error for the $k^{t h}$ user is equivalent to minimizing $\mathrm{E}\left\{\left\|\mathbf{r}_{k}-\hat{\mathbf{r}}_{k}\left(\mathbf{y}_{k}\right)\right\|^{2}\right\}$, where $\mathbf{y}_{k}$ is the matched filter (coherent version in our case) output in vector form for the $k^{\text {th }}$ user, it is a sufficient statistic for $\mathbf{r}_{k}$. The solution to this nonlinear MMSE estimation problem is the conditional mean estimate

$$
\begin{aligned}
& \hat{\mathbf{r}}_{k}=\mathrm{E}\left\{\mathbf{r}_{k} \mid \mathbf{y}_{k}\right\}=\sum_{m=1}^{M} b_{k, m} \mathbf{X}_{k, m} \mathbf{h}_{k} \\
& b_{k, m}=P\left[i_{k}(j)=m \mid \mathbf{y}_{k}\right]
\end{aligned}
$$

From the above equation, one can see that the MMSE estimate of $\mathbf{r}_{k}$ is a weighted sum of all the $M$ orthogonal signals. The interference canceled vector is obtained as

$$
\mathbf{r}_{k}^{\prime}=\mathbf{r}-\sum_{i \neq k} \hat{\mathbf{r}}_{i}=\mathbf{X}_{k} \mathbf{h}_{k}+\mathbf{r}_{c}+\mathbf{n}=\mathbf{X}_{k} \mathbf{h}_{k}+\mathbf{w}
$$

where $\mathbf{r}_{c}$ stands for cancellation residual, and the vector $\mathbf{w}$ is defined as $\mathbf{w}=\mathbf{r}_{c}+\mathbf{n}$. The $m^{\text {th }}$ element of $\mathbf{y}_{k}$, denoted by $y_{k, m}$ is formed by

$$
\begin{aligned}
& y_{k, m}=\left(\mathbf{X}_{k, m} \hat{\mathbf{h}}_{k}\right)^{*} \mathbf{r}_{k}^{\prime} \approx \\
& \begin{cases}\sum_{l=1}^{L_{k}}\left\|\mathbf{x}_{k, l, m} h_{k, l}\right\|^{2}+\sum_{l=1}^{L_{k}}\left(\mathbf{x}_{k, l, m} h_{k, l}\right)^{*} \mathbf{w} & \text { if } i_{k}(j)=m, \\
\sum_{l=1}^{L_{k}}\left(\mathbf{x}_{k, l, m} h_{k, l}\right)^{*} \mathbf{w} & \text { if } i_{k}(j) \neq m .\end{cases} \\
& = \begin{cases}N P_{k}+w & \text { if } i_{k}(j)=m, \\
w & \text { if } i_{k}(j) \neq m .\end{cases}
\end{aligned}
$$

where $P_{k}=\sum_{l=1}^{L_{k}}\left|h_{k, l}\right|^{2}$ stands for the instantaneous (and time-varying) received power for the $k^{\text {th }}$ user and $w=\sum_{l=1}^{L}\left(\mathbf{x}_{k, l, m} h_{k, l}\right)^{*} \mathbf{w}$ stands for the noise plus residual interference component in the decision statistic. Provided that the processing gain and the number of users are large, we can model $w$ as complex Gaussian random variable $w \sim \mathcal{C N}\left(0, \sigma_{w}^{2}\right)$. The approximation in (8) is due to the facts that perfect channel estimation is assumed so that $\hat{\mathbf{h}}_{k}=\mathbf{h}_{k}$ and that the autocorrelation of the same user's scrambling codes is assumed to approximate delta function so that the cross-correlation terms $\left(\mathbf{x}_{k, i, m} h_{k, i}\right)^{*} \mathbf{x}_{k, j, m} h_{k, j}, i \neq j$ approximate zero and can be omitted.

The conditional pdf is thus

$$
\begin{aligned}
& f\left(\mathbf{y}_{k} \mid i_{k}(j)=m\right) \\
& =\frac{1}{\left(\pi \sigma_{w}^{2}\right)^{M}} \exp \left[-\frac{\left|y_{k, m}-N P_{k}\right|^{2}}{\sigma_{w}^{2}}-\sum_{\substack{j=1 \\
j \neq m}}^{M} \frac{\left|y_{k, j}\right|^{2}}{\sigma_{w}^{2}}\right] \\
& =\frac{1}{\left(\pi \sigma_{w}^{2}\right)^{M}} \exp \left[\frac{-N^{2} P_{k}^{2}+2 N P_{k} \operatorname{Re}\left\{y_{k, m}\right\}}{\sigma_{w}^{2}}-\sum_{j=1}^{M} \frac{\left|y_{k, j}\right|^{2}}{\sigma_{w}^{2}}\right]
\end{aligned}
$$


Note that

$$
\begin{aligned}
& f\left[i_{k}(j)=m \mid \mathbf{y}_{k}\right]=\frac{f\left[\mathbf{y}_{k} \mid i_{k}(j)=m\right] \cdot P\left[i_{k}(j)=m\right]}{f\left[\mathbf{y}_{k}\right]} \\
& f\left[\mathbf{y}_{k}\right]=\sum_{q=1}^{M} P\left[i_{k}(j)=q\right] \cdot f\left[\mathbf{y}_{k} \mid i_{k}(j)=q\right] \\
& P\left[i_{k}(j)=1\right]=\cdots=P\left[i_{k}(j)=M\right]=\frac{1}{M}
\end{aligned}
$$

and combine the above equations with (10), yielding

$$
\begin{aligned}
b_{k, m} & =P\left[i_{k}(j)=m \mid \mathbf{y}_{k}\right]=\frac{f\left[\mathbf{y}_{k} \mid i_{k}(j)=m\right]}{\sum_{q=1}^{M} f\left[\mathbf{y}_{k} \mid i_{k}(j)=q\right]} \\
& =\frac{\exp \left[-\frac{N^{2} P_{k}^{2}}{\sigma_{w}^{2}}\right] \cdot \exp \left[\frac{2 N P_{k} \operatorname{Re}\left\{y_{k, m}\right\}}{\sigma_{w}^{2}}\right]}{\exp \left[-\frac{N^{2} P_{k}^{2}}{\sigma_{w}^{2}}\right] \cdot \sum_{q=1}^{M} \exp \left[\frac{2 N P_{k} \operatorname{Re}\left\{y_{k, q}\right\}}{\sigma_{w}^{2}}\right]} \\
& =\frac{\exp \left[2 N P_{k} \operatorname{Re}\left\{y_{k, m}\right\} / \sigma_{w}^{2}\right]}{\sum_{q=1}^{M} \exp \left[2 N P_{k} \operatorname{Re}\left\{y_{k, q}\right\} / \sigma_{w}^{2}\right]}
\end{aligned}
$$

If $\mathbf{r}_{c}=\mathbf{0}$, i.e., the cancellation residual is neglected by assuming perfect cancellation, the noise plus interference variance can be approximated as $\sigma_{w}^{2} \approx \sigma_{n}^{2}=$ $N \sum_{l=1}^{L_{k}}\left|h_{k, l}\right|^{2} N_{0}=P_{k} N N_{0}$, leading to the solution

$$
\begin{aligned}
b_{k, m} & =\frac{\exp \left[\frac{2 N P_{k} \operatorname{Re}\left\{y_{k, m}\right\}}{\sigma_{n}^{2}}\right]}{\sum_{q=1}^{M} \exp \left[\frac{2 N P_{k} \operatorname{Re}\left\{y_{k, q}\right\}}{\sigma_{n}^{2}}\right]}=\frac{\exp \left[\frac{2 N P_{k} \operatorname{Re}\left\{y_{k, m}\right\}}{P_{k} N N_{0}}\right]}{\sum_{q=1}^{M} \exp \left[\frac{2 N P_{k} \operatorname{Re}\left\{y_{k, q}\right\}}{P_{k} N N_{0}}\right.} \\
& =\frac{\exp \left[2 \operatorname{Re}\left\{y_{k, m}\right\} / N_{0}\right]}{\sum_{q=1}^{M} \exp \left[2 \operatorname{Re}\left\{y_{k, q}\right\} / N_{0}\right]}
\end{aligned}
$$

Unfortunately, we found out that direct implementation of (12) leads to a numerically unstable algorithm, which was not pointed out in [4]. The problem can be tackled by performing the MMSE estimation in the log domain, similar to the idea presented in [8] for iterative decoding. With Log-NMIC algorithm, the coefficient $b_{k, m}$ associated with each orthogonal component is calculated as

$$
b_{k, m}=e^{\frac{2 \operatorname{Re}\left\{y_{k, m}\right\}}{N_{0}}-\max ^{*}\left(\frac{2 \operatorname{Re}\left\{y_{k, 1}\right\}}{N_{0}}, \cdots, \frac{2 \operatorname{Re}\left\{y_{k, M}\right\}}{N_{0}}\right)}
$$

where the function $\max ^{*}()$ is defined as

$$
\max ^{*}(x, y)=\ln \left(e^{x}+e^{y}\right)=\max (x, y)+\ln \left(1+e^{-|x-y|}\right)
$$

which is max operation compensated with a correction term $\ln \left(1+e^{-|x-y|}\right)$. Also

$$
\max ^{*}(x, y, z)=\max ^{*}\left[\max ^{*}(x, y), z\right]
$$

The NMIC algorithm can be expressed as

$$
\begin{aligned}
& \hat{\mathbf{r}}_{i}=\mathrm{E}\left\{\mathbf{r}_{i} \mid \mathbf{y}_{i}\right\}=\sum_{m=1}^{M} b_{i, m} \mathbf{X}_{i, m} \mathbf{h}_{i} ; \quad \mathbf{r}_{k}^{\prime}=\mathbf{r}-\sum_{i \neq k} \hat{\mathbf{r}}_{i} ; \\
& z_{k}^{\mathrm{NMIC}}(m)=\operatorname{Re}\left\{\mathbf{y}_{k}\right\}=\operatorname{Re}\left\{\hat{\mathbf{h}}_{k}^{*} \mathbf{X}_{k, m}^{*} \mathbf{r}_{k}^{\prime}\right\}
\end{aligned}
$$

The above scheme assumes perfect cancellation and zero cross-correlation which is not the case in practice and leads to suboptimal solution. Now, we derive an adaptive algorithm [4] that takes the imperfect cancellation and non-zero cross-correlation into account and adaptively estimates the noise plus interference variance $\sigma_{w}^{2}$. We know that

$$
\mathrm{E}\left[\left\|\mathbf{y}_{k}\right\|^{2}\right]=\sum_{q=1}^{M} \mathrm{E}\left[\left|y_{k, q}\right|^{2}\right]=N^{2} \mathrm{E}\left[P_{k}^{2}\right]+M \sigma_{w}^{2}
$$

Therefore

$$
\sigma_{w}^{2}=\frac{\mathrm{E}\left[\left\|\mathbf{y}_{k}\right\|^{2}\right]-N^{2} \mathrm{E}\left[P_{k}^{2}\right]}{M}
$$

The performance of NMIC algorithm can be improved if $\sigma_{n}^{2}$ in (12) is replaced by $\hat{\sigma}_{w}^{2}$, which is adaptively estimated by averaging $\frac{\left\|\mathbf{y}_{k}\right\|^{2}-N^{2} P_{k}^{2}}{M}$ over the whole block of symbols. This leads to adaptive Log-NMIC algorithm

$$
b_{k, m}=e^{\frac{2 \operatorname{Re}\left\{y_{k, m}\right\}}{\hat{\sigma}_{w}^{2} / N P_{k}}-\max *\left(\frac{2 \operatorname{Re}\left\{y_{k, 1}\right\}}{\hat{\sigma}_{w}^{2} / N P_{k}}, \cdots, \frac{2 \operatorname{Re}\left\{y_{k, M}\right\}}{\hat{\sigma}_{w}^{2} / N P_{k}}\right)}
$$

\section{Soft channel estimation}

The soft linear MMSE estimate (approximate version) of $\mathbf{h}$ is formulated as

$$
\hat{\mathbf{h}}_{\text {soft }}=\hat{\mathbf{P}}^{*} \mathrm{E}\left[\mathbf{A}^{*} \mid \mathbf{r}\right]\left(\mathrm{E}[\mathbf{A} \mid \mathbf{r}] \hat{\mathbf{P}} \mathrm{E}\left[\mathbf{A}^{*} \mid \mathbf{r}\right]+N_{0} \mathbf{I}\right)^{-1} \mathbf{r}
$$

where $\mathbf{P}=\mathrm{E}\left[\mathbf{h h}^{*}\right]=\operatorname{diag}\left(\bar{P}_{1,1}, \bar{P}_{1,2}, \cdots, \bar{P}_{k, l}, \cdots, \bar{P}_{K, L_{K}}\right)$, and $\bar{P}_{k, l}$ is the average received power from the $k^{t h}$ user's $l^{\text {th }}$ path. $\mathrm{E}[\mathbf{A} \mid \mathbf{r}]$ is soft estimate of $\mathbf{A}$ derived based on (6) for MAP-PIC as explained in Section III-A. For the NMIC algorithm, we replace (6) with $\mathrm{E}\left[\mathbf{w}_{k}^{n}(j) \mid \mathbf{r}\right]=$ $\sum_{m=1}^{M} b_{k, m} \mathbf{w}_{m}^{n}$. The rest of the derivation is the same.

If $\mathrm{E}[\mathbf{A} \mid \mathbf{r}]$ is not available, we replace it with $\hat{\mathbf{A}}$ in equation (16), leading to the hard version of the LMMSE channel estimator which is used in the conventional PIC

$$
\hat{\mathbf{h}}_{\text {hard }}=\hat{\mathbf{P}}^{*} \hat{\mathbf{A}}^{*}\left(\hat{\mathbf{A}} \hat{\mathbf{P}} \hat{\mathbf{A}}^{*}+N_{0} \mathbf{I}\right)^{-1} \mathbf{r}
$$

\section{Numerical Results}

In our simulations, each user transmits one of $M=8$ Walsh codes spread to a total length of $N=64$ chips. The effective spreading of the system is $N / \log _{2} M=64 / 3$ chips per bit. Different users are separated by different scrambling codes $\mathbf{C}_{k}(j)$ which are random, and differ from symbol to symbol. Channels are independent Rayleigh fading channels with the classical "bath tub" power spectrum. That is, the channel gain $h_{k, l}(t)$ is a complex circular Gaussian process with autocorrelation function $\mathrm{E}\left[h_{k, l}^{*}(t) h_{k, l}(t+\tau)\right]=P_{k, l} J_{0}\left(2 \pi f_{d} \tau\right)$ where $f_{d}$ is the maximum Doppler frequency, $J_{0}(x)$ is the zeroth order Bessel function of the first kind. The Doppler shifts on each of the multipath components are due to the relative motion between the base station and mobile units. Here, the normalized Doppler frequency is assumed to be $f_{d} T=0.01$. The number of users is $K=21$, which means a fully loaded system. The simulation results are averaged over random distributions of fading, noise, delay, and scrambling code through numerous Monte-Carlo runs. 


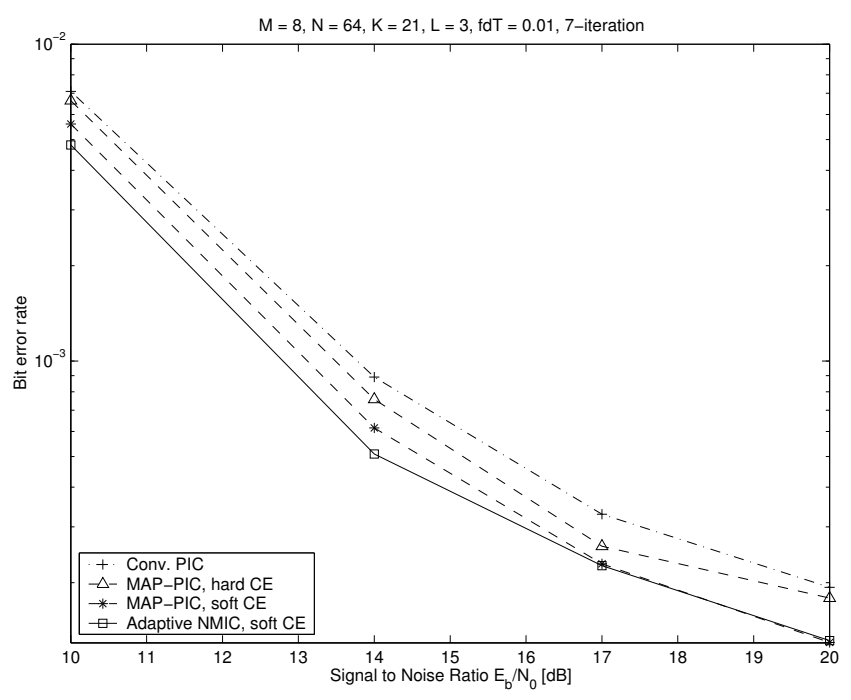

Fig. 2. Performance of different IC schemes. The curves are plotted for the $7^{t h}$ stage IC.

For simplicity, the simulated system is assumed to be chip-synchronous, i.e., all path delays are assumed to be multiples of $T_{c}$. However, the system is asynchronous on the symbol level. Perfect slow power control is assumed in the sense that $\bar{P}_{k}=\sum_{l=1}^{L_{k}} \bar{P}_{k, l}$, the average received power, is equal for all users. The channel coefficients are normalized so that each user has unity gain, i.e., $\bar{P}_{k}=$ $\sum_{l=1}^{L_{k}} \bar{P}_{k, l}=1$. The number of multipath channels $L_{k}$ is set to be $3,\left(L_{k}=L=3\right)$ for all $k$.

The noncoherent matched filter [3] is used for the first stage of the PIC scheme to account for the fact that channel estimates are not yet available at the initial stage. In the following stages, both interference cancellation and channel estimation are carried out in decision directed mode using the detected data from the previous iteration.

The conventional PIC and the proposed soft PIC algorithms are compared numerically in Fig. 2. As indicated by the figure, the soft cancellation alone yields limited performance improvement, while the gain is much more obvious when the soft channel estimation is performed in conjunction with soft cancellation. Among the two soft PIC algorithms, the adaptive Log-NMIC performs slightly better than the MAP-PIC at low SNR; however, their performance is essentially the same in high SNR region, both achieve up to a $2 \mathrm{~dB}$ gain compared to the conventional PIC in a 21-user system. The performance of non-adaptive NMIC is inferior to adaptive NMIC as well as MAP-PIC, and is not shown here.

Fig. 3 shows the convergence property of the conventional and soft PIC scheme (the MAP version). They both take 6 or 7 iterations to converge. The gain at each iteration becomes bigger as the SNR increases; the gap between them increases as the iteration goes on. The conclusion is that we need to perform enough stages and maintain a sufficiently high SNR to be able to fully benefit from the soft cancellation and soft channel estimation processes.

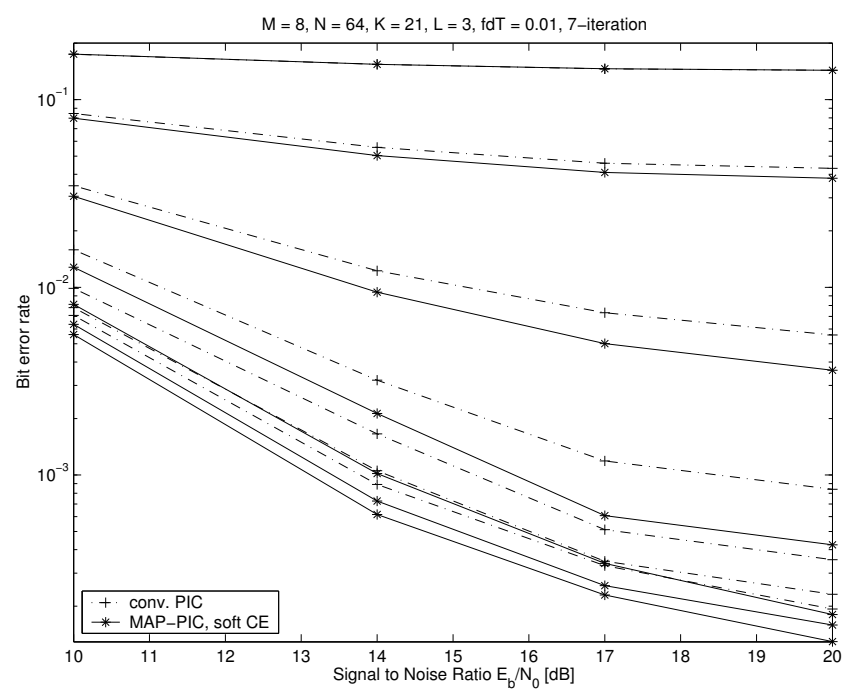

Fig. 3. Convergence property of IC schemes. Topmost curve represents the noncoherent first stage and the second curve from top represents the first stage IC, the bottommost curve represents the $7^{t h}$ stage IC.

\section{Conclusions}

In this paper, two soft cancellation schemes, one based on the MAP criterion, the other based on the nonlinear MMSE estimation are presented for an orthogonal modulated asynchronous DS-CDMA system without convolutional coding. Simulation results show that they achieve superior performance compared to the conventional PIC using hard decision feedback and that the soft information should be used for both interference cancellation and channel estimation in order to achieve the utmost performance.

\section{REFERENCES}

[1] M. Kawabe, T. Sato, T. Kato, A. Fukasawa, and R. Kohno. Mary CDMA scheme based on interference cancellation Proc. Asia Pacific Conference on Circuit and Systems, pages 33-38, 1994.

[2] J. Kim, S. Yoon, S. Kang and C. Kang. Interference cancellation technique using channel parameter estimation in DS/CDMA system with M-ary orthogonal modulation. Electronics Letters, 34(12):1194-1195, June 1998.

[3] E. Ström, S. Miller. "Iterative demodulation and channel estimation of orthogonal signalling formats in asynchronous DS-CDMA systems". IEICE Transactions on Electronics, vol. E85-C, no. 3, pp. 442-451, March, 2002.

[4] S. Gollamudi, S. Ngaraj, Y. Huang, R. Buehrer. "Optimal multistage interference cancellation for CDMA systems using the nonlinear MMSE criterion." Proc. the 32nd Asilomar Conference on Signals, Systems, and Computers, pp. 665-669, Nov. 1998.

[5] H. Gamal, E. Geraniotis. "Iterative multiuser detection for coded CDMA signals in AWGN and fading channels". IEEE Journal on Selected Areas in Communications, vol. 18, no. 1, pp. 30-41, Jan. 2000.

[6] X. Wang, H. Poor. "Iterative (Turbo) soft interference cancellation and decoding for coded CDMA". Transactions on Communications, vol. 47, no. 7, pp. 1046-1061, July 1999.

[7] J. Hagenauer, E. Offer, L. Papke. "Iterative decoding of binary block and convolutional codes". IEEE Transactions on Information Theory, vol. 42, no. 2, pp. 429-445, March 1996.

[8] P. Robertson, E. Villebrun, P. Hoeher. "A comparison of optimal and sub-optimal MAP decoding algorithms operating in the log domain." Proc. International Conference on Communications, vol. 2, pp. 1009-1013, June 1995. 